Ann. Zootech., I977, 26 (I), II3-II8.

Note

\title{
Transit digestif chez le poulet de 5 semaines Recherche d'une variation entre familles de pères
}

\author{
J. P. LAPLACE \\ avec la collaboration technique de C. GERMarN \\ Laboratoire de Physiologie de la Nutrition, \\ Centre national de Recherches zootechniques, I.N.R.A., \\ $7835^{\circ}$ Jouy-en-Josas (France)
}

\begin{abstract}
Résumé
Le transit digestif d'une dose unique de ${ }^{141} \mathrm{Ce}$ a été étudié chez I 55 coquelets âgés de 5 semaines, issus de mères génétiquement comparables, mais de 4 pères distincts, par abattage à 5 stades entre la $30^{\circ}$ et la $I_{5} \mathrm{O}^{e} \mathrm{mn}$ après l'administration du marqueur radioactif. Il n'est pas possible de mettre en évidence un effet de la famille du père à l'égard des caractéristiques du transit digestif chez $c \in s$ sujets jeunes dans les conditions expérimentales utilisées.
\end{abstract}

\section{Introduction}

Des travaux relativement nombreux ont été consacrés au passage des matériaux alimentaires dans le tube digestif des oiseaux. La synthèse apportée par STURKIE (I965) et par ZiSWILER et FARNER (I972) montre combien le temps de passage est variable en fonction des caractéristiques physiques, chimiques et nutritionnelles de l'alimentation. Le transit est aussi d'autant plus bref que l'on considère des sujets plus jeunes. Ainsi l'oxyde de chrome lié aux particules alimentaires solides peut être retrouvé dans les fèces après $2 \mathrm{~h} 30 \mathrm{mn}$ et sa quasitotalité récupérée en $24 \mathrm{~h}$; de même $5^{\circ} \mathrm{p}$. Ioo du baryum radioactif administré à des poussins sont excrétés en 4 à 5 heures. Cependant peu de travaux ont été consacrés à l'étude d'influences d'ordre génétique. L'observation d'importantes 
différences d'efficacité alimentaire, notamment chez des poules pondeuses, pose la question de l'origine de ces variations, dans la perspective d'une éventuelle sélection de lignées divergentes au sein d'une même souche.

\section{Matériel et méthodes}

Dans cet esprit, le présent travail a été consacré à la recherche d'une variation entre familles de pères au niveau du transit digestif chez I55 coquelets âgés de 5 semaines. Ces animaux proviennent du cheptel de la Station de Génétique factorielle et appartiennent à 4 groupes :

Groupe $I=25$ coquelets de 4 mères et père $A$.

Groupe $2=40$ coquelets de 7 mères et père $B$.

Groupe $3=45$ coquelets de 7 mères et père $C$.

Groupe $4=45$ coquelets de 7 mères et père $D$.

Les mères utilisées pour les 4 groupes appartiennent au cheptel expérimental de la Station et sont génétiquement comparables. Les 4 pères distincts, croisés Wyandotte $\times$ Rhode Island, ont été pris au hasard parmi plusieurs centaines d'animaux de l'élevage du Magneraud; leur pédigree exact est inconnu.

Les I55 coquelets ont été obtenus en 5 éclosions successives échelonnées du 28 janvier au 25 février I976. Ils ont été utilisés expérimentalement entre le 3 et le 3 I mars I976. Ils ont disposé, en cage individuelle, d'eau et d'aliment à volonté. L'aliment, distribué sous forme de farine, est d'une formulation standard : maïs, 69,7 - tourteau de soja, I5 - tourteau de tournesol, ro - poudre d'os, 2,5 - calcimarine, I - sel vétérinaire, 0,3 - complément minéral et vitaminique, $\mathrm{r}, 5$ - avec en sus : anticoccidien, 0,05 et antioxydant, 0,00I25. Les conditions d'ambiance sont celles d'une alternance jour-nuit, $8 \mathrm{~h}-20 \mathrm{~h}$, en éclairage artificiel, et d'une température de $22{ }^{\circ} \mathrm{C}$.

L'expérimentation a comporté l'administration d'une dose unique de ${ }^{141} \mathrm{Ce}$, par intubation directe de la solution chlorhydrique, sous un volume de $0,1 \mathrm{ml}$ représentant une dose de 5 à 6 microcuries. L'intubation est systématiquement effectuée à $9 \mathrm{~h}$. Les coquelets sont ensuite sacrifiés à 5 stades après administration du marqueur (30-60-90-120 et $150 \mathrm{mn}$ ) à raison de 5 sujets de père $\mathrm{A}, 8$ sujets de père $B$ et 9 sujets de père $C$ ou $D$ par stade. Comme pour l'administration du ${ }^{141} \mathrm{Ce}$, la méthodologie simplifiée mise en œuvre chez le lapin (LAPLACE et LEBAS, I975) pour les comptages a été appliquée intégralement aux fèces collectées entre l'intubation et l'abattage, et aux 6 compartiments digestifs ci-après : jabot, proventricule, gésier, duodénum et anse de Meckel (intestin grêle), cæca, système colorecto-cloaqual (côlon).

\section{Résultats et discussion}

Les quantités moyennes de radioactivité retrouvées dans les divers compartiments digestifs ainsi définis et dans les fèces, aux 5 stades d'abattage et pour chacun des 4 groupes de coquelets sont présentées dans la figure I. Aucune diffé- 

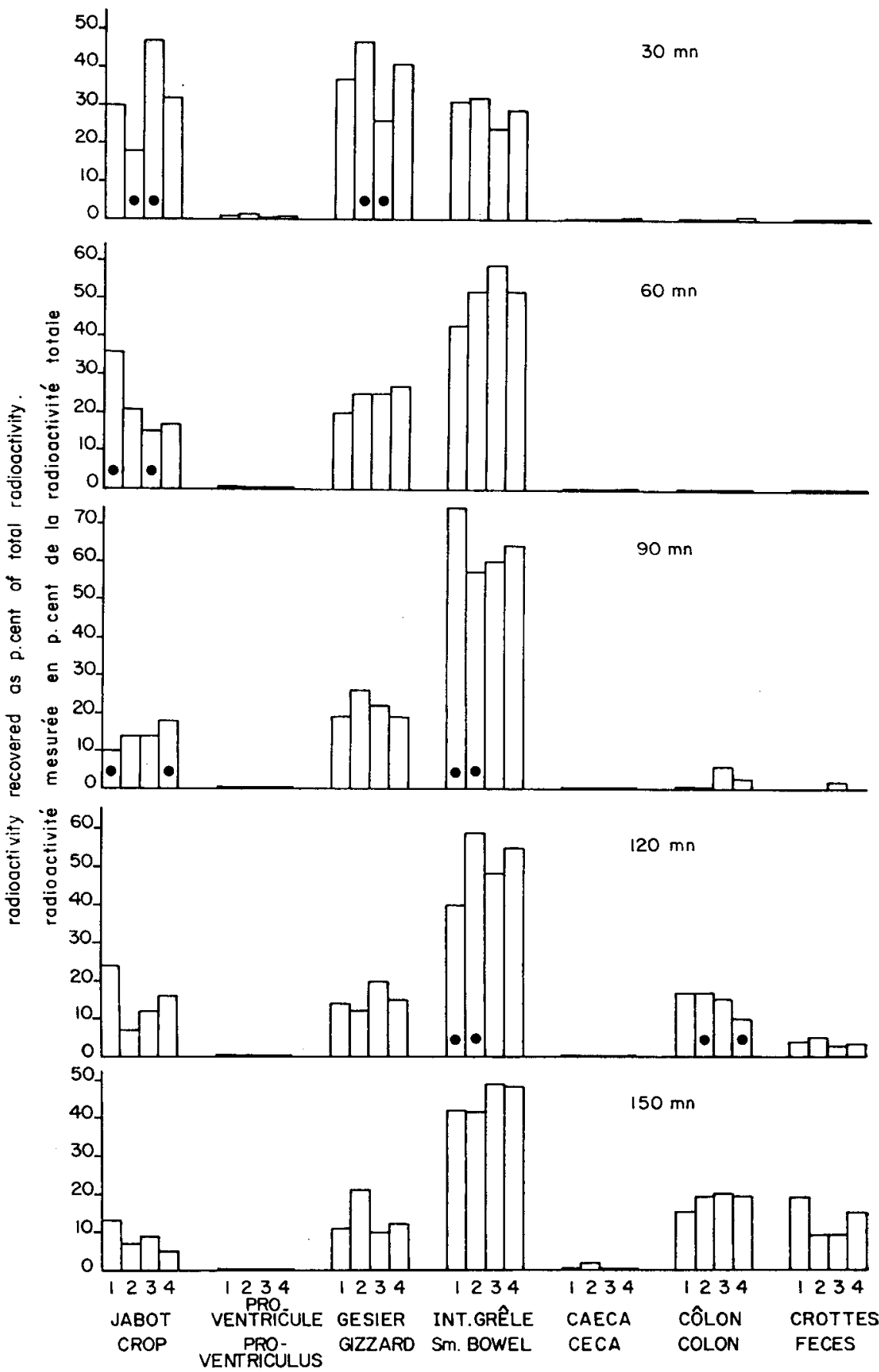

FIG. I. - Radioactivité moyenne retrouvée (c.p.m.) dans les divers compartiments digestifs et les fèces au terme de délais variables après intubation de ${ }^{141} \mathrm{Ce}$ en solution chlorhydrique chez des coquelets de 5 semaines de 4 origines paternelles distinctes (groupes I à 4 ). Les colonnes marquées d'I point différent entre elles (comparaisons de moyennes 2 à 2 pour un même stade et un même compartiment) au seuil 5 p. cent.

Mean radioactivity (c.p.m.) remaining within the various digestive compartments and found in the faeces at various time after the administration of ${ }^{141} \mathrm{Ce}$ (acid solution) to 5 week-old cockerels owing to 4 paternities (groups $\mathrm{I}$ to 4 ). The columns bearing a point significantly differ ( 5 p. cent) between them (comparisons of the means at the same time and for the same compartment. 


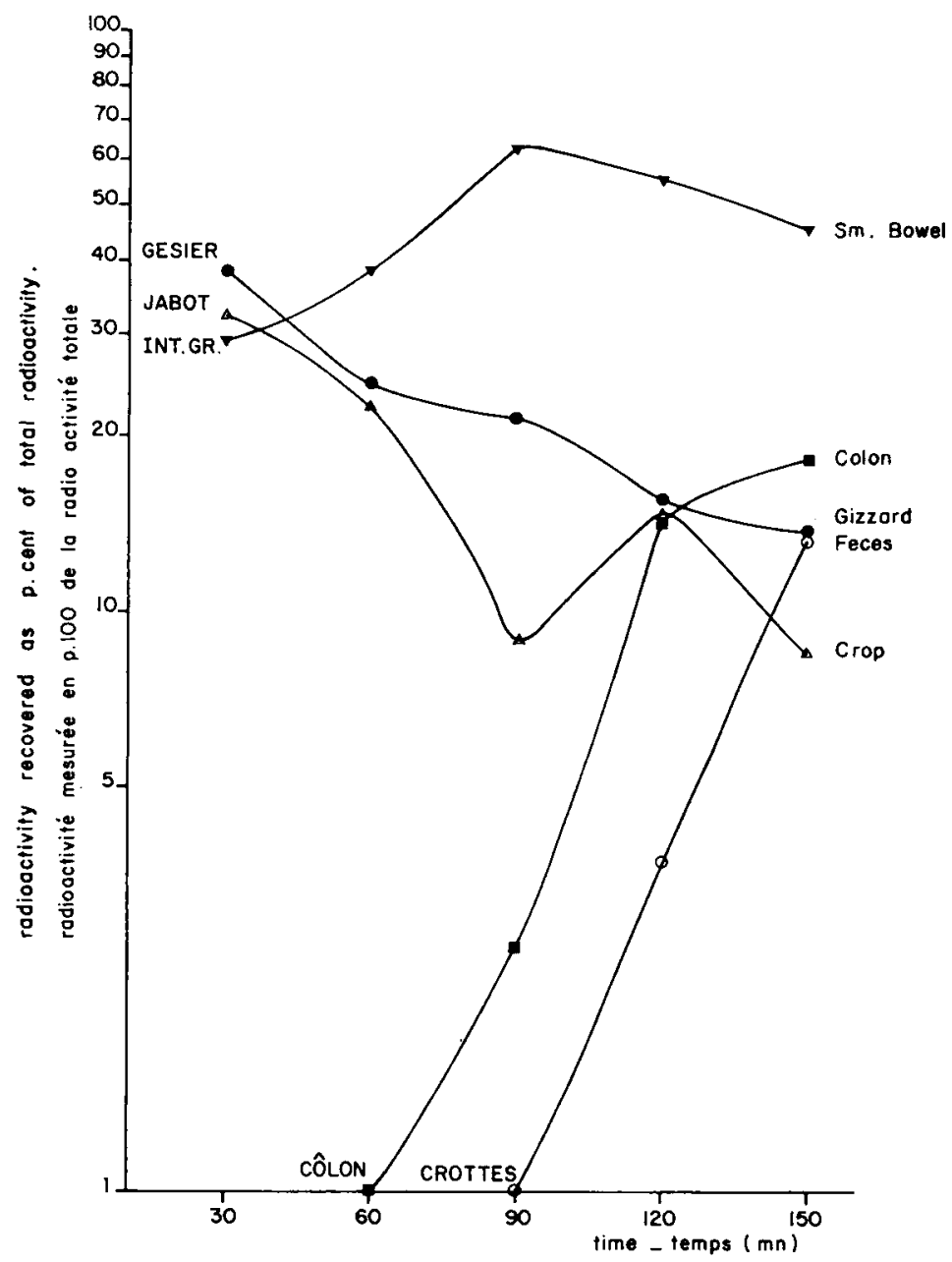

FIG. 2. - Evolution en fonction du temps de la quantité moyenne de vadioactivité présente dans le jabot, le gésier, l'intestin grêle (duodénum et anse de Meckel). le côlon (système colo-recto-cloaqual non compris les caca) et les fèces, après intubation d'une dose unique de ${ }^{141} \mathrm{Ce}$ chez ${ }^{1} 55$ coquelets âgés de 5 semaines. Chaque point correspond à la moyenne de $3^{I}$ résultats individuels.

Evolution of the mean radioactivity found in the crop, the gizzard, the small bowel (duodenum and Meckel's loop), the colon (colon, rectum and cloaca but caeca excluded) and the feces during I $50 \mathrm{mn}$ after the administrition of the single dose of ${ }^{141} \mathrm{Ce}$ to I 55 cockevels (5 week-old). Each point is the mean of 31 individual values.

rence systématique entre groupes, liée à un effet de la famille du père ne peut être mise en évidence. Seules des différences occasionnelles entre moyennes comparées 2 à 2 pour un même compartiment et un même stade peuvent être observées.

Le groupement des données recueillies pour les 4 groupes permet d'établir les cinétiques d'apparition-disparition des quantités de radioactivité pour chaque compartiment considéré, sur la base de la moyenne des résultats de 3 I coquelets par stade d'abattage (fig. 2). On constate ainsi que, dans les conditions utilisées 
d'alimentation et d'administration du marqueur, la radioactivité est, dès le stade $30 \mathrm{mn}$, répartie dans le jabot (32 p. I0o), le gésier (38 p. Ioo) et l'intestin grêle (29 p. I00). Au niveau des portions proximales du tube digestif, le proventricule n'est à aucun stade le siège d'une accumulation de radioactivité (maximum I p. IOo). L'anse de Meckel est franchie par le front de radioactivité avant la $90^{\mathbf{e}}$ minute, puisque le côlon en renferme à ce stade près de 3 p. ıoo. Ce segment digestif postérieur est rapidement traversé, et près de $4 \mathrm{p}$. Ioo de la radioactivité est retrouvée dans les fèces $120 \mathrm{mn}$ après administration. Les cæca, dont l'état de réplétion par du contenu digestif a été constamment observé lors des abattages, ne contiennent à aucun stade une quantité notable de radioactivité (maximum o,58 p. roo). Ce fait particulier peut être rattaché à l'intermittence de l'activité motrice cæcale (FARGeAs et al., I963; Roche, I974) compte tenu de l'horaire préois adopté par rapport aux rythmes d'éclairement et de prise de nourriture spontanée par exemple. Enfin, le réhaussement au stade $\mathbf{2 0} \mathrm{mn}$ du profil de décroissance de la radioactivité dans le jabot correspond à l'ampleur des différences entre animaux, les données relatives à chaque stade étant obtenues sur des coquelets nécessairement différents. Au demeurant, ces mêmes variations individuelles sont susceptibles de rendre compte des quelques différences occasionnellement constatées entre groupes de père différent.

Compte tenu des limites de temps de l'expérimentation, il n'est pas possible d'évaluer le temps de rétention dans le tube digestif de nos animaux. Toutefois nos résultats pour des coquelets de 5 semaines s'avèrent compatibles avec ceux de SkLAN et al. (I975) obtenus chez des sujets d'âge compris entre 6 et 9 semaines, avec le même radio isotope. Ces auteurs confirment en effet l'augmentation du temps de passage des aliments avec l'âge, et apportent des valeurs d'une totale fiabilité (ingestion d'aliment uniformément marqué - équilibre de marquage dans le tube digestif - comptages après incinération et reprise en milieu chlorhydrique). Aussi la méthodologie simplifiée mise en œuvre dans cet essai d'orientation ne peut-elle rendre compte de l'absence d'influence paternelle dans nos conditions expérimentales.

En conclusion, au terme de cette expérimentation, il n'est pas possible de mettre en évidence un effet de la famille du père à l'égard des caractéristiques du transit digestif chez le poulet de 5 semaines. Néanmoins cette indication devra être complétée par d'autres travaux impliquant des pères de pédigrees connus et très différents, et être renouvelée sur des sujets plus âgés.

Ręu poù publication en décembre 1976.

\section{Summary}

Digestive transit in 5 weeks old chicken: search for a variation between sire families

The digestive transit of a single dose of ${ }^{141} \mathrm{Ce}$ was studied in 155 five weeks old cockerels from genetically related dams, but from 4 different sires, by slaughtering the animals at 5 stages between the 3 oth and the $I_{5}$ oth $\mathrm{min}$ after administration of the radioactive tracer. It was not possible to $\epsilon$ vidence any effect of the sire family on the characteristics of the digestive transit in these young subjects and under the present experimental conditions. 


\section{Références bibliographiques}

Fargeas M. J., Fargeas J., Le Bars H., Sevrez Cl., I963. Étude de la motricité cæcale, chez la Poule, par la technique de la fistule permanente. Rev. Med. Vet., 114, 693-707.

Laplace J. P., Lebas F., I975. Le transit digestif chez le lapin. 3) Influence de l'heure et du mode d'administration sur l'excrétion fécale du Cérium I 4I, chez le lapin alimenté ad libitum. Ann. Zootech., 24, 255-265.

Roche M., I974. Motricité gastro-intestinale chez le Poulet. Ann. Rech. Vet., 5, 295-309.

Sklan D., Dubrov D., Eisner U., Hurwitz S., 1975. ${ }^{51} \mathrm{Cr}$-EDTA, ${ }^{91} \mathrm{Y}$ and ${ }^{141} \mathrm{Ce}$ as Nonabsorbed reference substances in the gastro intestinal tract of the chicken. $J . N u t r ., 105,1_{549-}$ $155^{2}$.

Sturkie P. D., I965. Alimentary Canal : Anatomy, prehension, deglutition, appetite, passage of ingesta, motility (chap. Io) in : Avian Physiology, $2^{\text {nd }}$ Ed., I Vol., 766 p., Cornell University Press Ed., Ithaca.

Zrswiler V., Farner D. S., 1972. Digestion and the digestive system (chap. 6), in Farner D. S. et KIng J. R., Avian Biology, Vol. Il, Academic Press Ed. 